\title{
Cervical Origin of the Right Subclavian Artery with a Nonbifurcating Left Cervical Carotid Artery
}

\author{
Mohamad Ezzeldina $^{a} \quad$ Eslam W. Youssef ${ }^{b} \quad$ Vibhav Bansalc \\ Ali Sultan Qurraie ${ }^{a}$ Osama Zaidat ${ }^{a}$ \\ a Interventional Neurology, Mercy Health St. Vincent Medical Center, Toledo, OH, USA; \\ ${ }^{\mathrm{b}}$ Radiology, University of Toledo, Toledo, OH, USA; ' Interventional Neurology, \\ Mercy Health St. Rita's Medical Center, Lima, OH, USA
}

\author{
Keywords \\ Congenital heart disease $\cdot$ Angiography $\cdot$ Subclavian artery $\cdot$ Internal carotid artery
}

A 55-year-old female with no significant past medical history sustained multiple traumatic injuries from a motor vehicle accident. During trauma workup, computed tomography of the head was unremarkable. However, the left internal carotid artery (ICA) could not be visualized on computed tomography angiography. There was concern for left ICA traumatic dissection.

Conventional cervical and cerebral angiography revealed a cervical origin of the right subclavian artery (CORSA). There was a nonbifurcating left cervical carotid artery with a single artery supplying all normal branches of the external carotid artery. The left middle cerebral artery was filled via a large left posterior cerebral artery. The left anterior cerebral artery was filled from the contralateral anterior cerebral artery via the anterior communicating artery. Interestingly, both ophthalmic arteries were supplied exclusively from the external carotid arteries (Fig. 1-4).

CORSA is a rare congenital anomaly that occurs when there is absence of the right fourth pharyngeal arch artery accompanied by persistence of the right carotid duct. In this setting,

Guarantor of Submission: The corresponding author is the guarantor of submission. 
Interventional

Neurology

Fig. 1. Arch aortogram showing the origin of the brachiocephalic artery and the left external carotid artery. The right brachiocephalic artery (black arrow) seen ascending high above the clavicle giving rise to the right subclavian artery (red arrow) that is travelling back caudally to enter the arm and a short segment of the right common carotid artery (blue arrow).

Fig. 2. Nonbifurcating left cervical carotid artery (green arrow). A single artery ascends supplying all normal branches of the external carotid artery.

\begin{tabular}{|c|c|}
\hline Intervent Neurol 2018;7:3 & \\
\hline DOI: 10.1159/000489019 & $\begin{array}{l}\text { (c) } 2018 \text { S. Karger AG, Basel } \\
\text { www.karger.com/ine }\end{array}$ \\
\hline
\end{tabular}

Ezzeldin et al.: CORSA
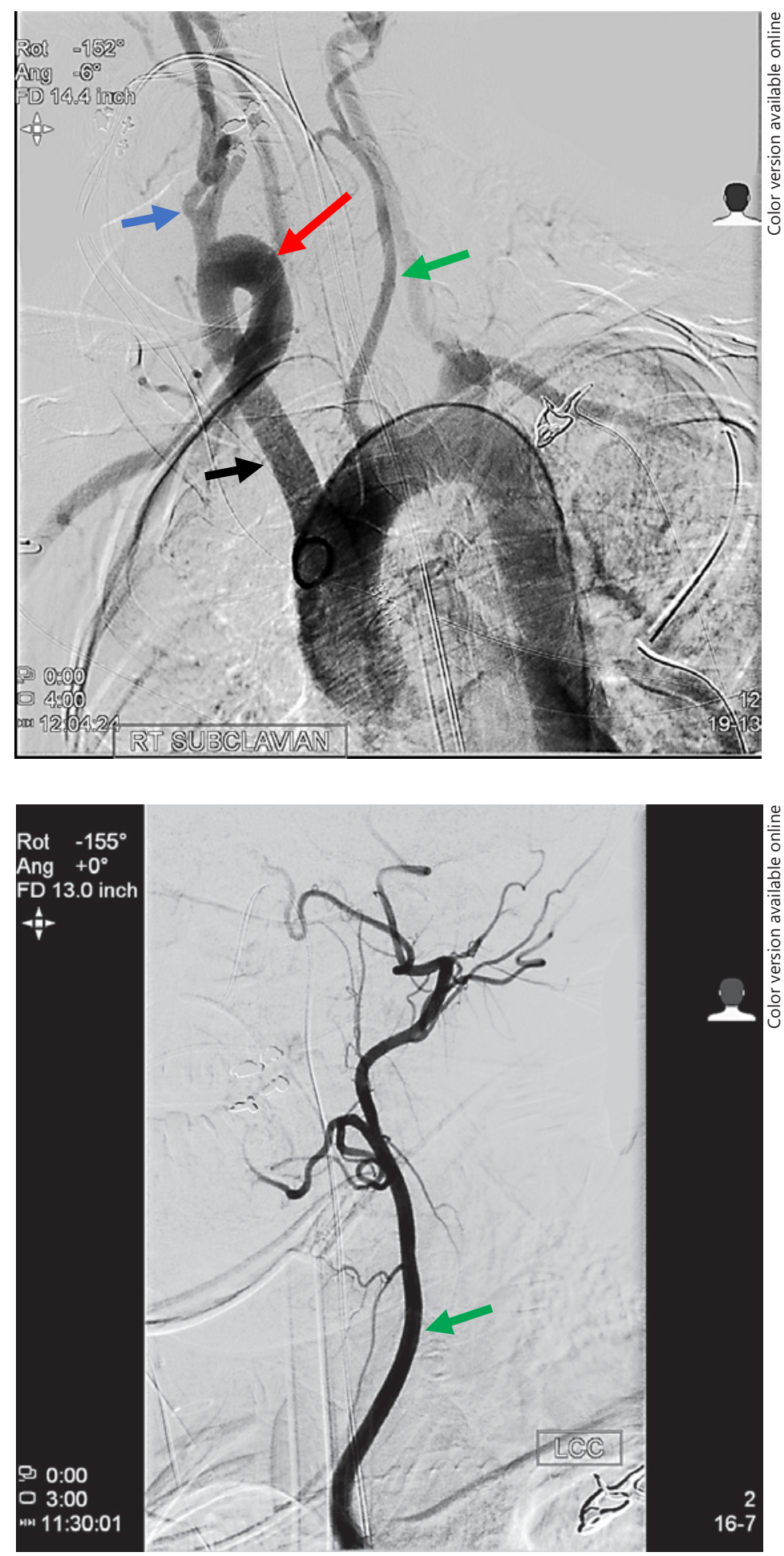

the seventh intersegmental artery joins the carotid duct, and the right subclavian artery originates from the right common carotid at the cervical origin (CORSA) [1].

Absence of the ICA is another rare congenital anomaly that can be unilateral or bilateral. Unilateral anomaly could be secondary to intrauterine mechanical and hemodynamic stresses with constriction by the amniotic bands. During normal embryogenesis, the ICA arises from 
Interventional

Neurology

Fig. 3. Right brachiocephalic artery injection showing the bifurcation of the brachiocephalic artery into the right subclavian artery (black arrow) and a short segment of the right common carotid artery (red arrow) shortly bifurcating into the right internal carotid artery and external carotid artery. Note the loops at the origin of both the internal carotid artery and external carotid artery (blue arrow).

Fig. 4. CT scan of the brain. Bone window showing the normal right carotid canal (red arrow) and the absent left carotid canal (blue arrow).

\begin{tabular}{l|l}
\hline Intervent Neurol 2018;7:399-402 \\
\hline DOI: 10.1159/000489019 & $\begin{array}{l}\text { c) 2018 S. Karger AG, Basel } \\
\text { www.karger.com/ine }\end{array}$ \\
\hline
\end{tabular}
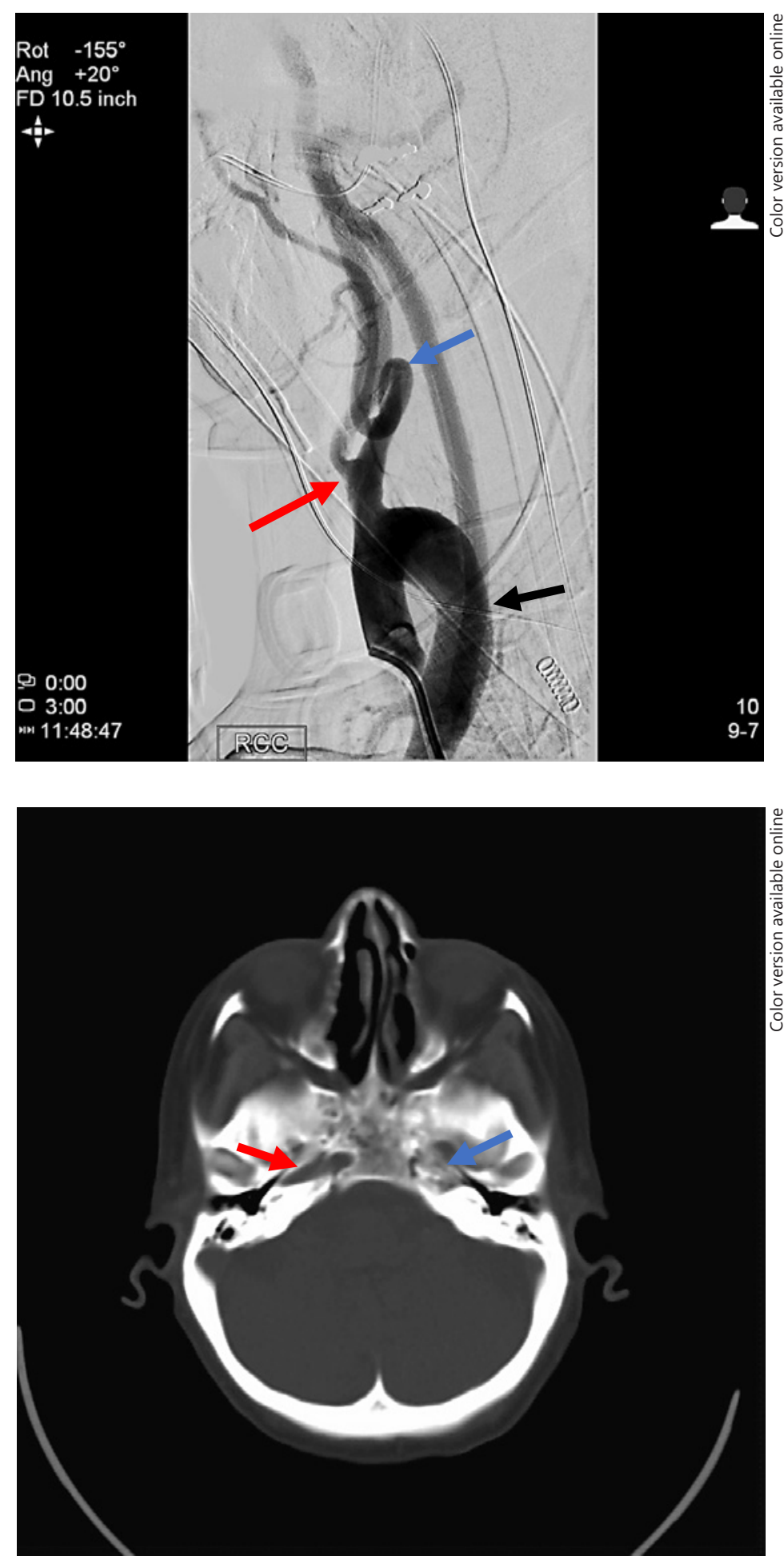

the third arch artery and the dorsal aorta. Agenesis of the ICA depends on the abnormal regression of the first and third aortic arches [2].

Despite their rather benign courses, these anomalies may have serious implications in certain settings. For instance, patients with agenesis of the ICA may be especially vulnerable to posterior circulation insufficiency as they may be at a much higher risk for simultaneous 
anterior circulation compromise. In the present case, traumatic dissection was suspected; however, the anomaly was delineated on computed tomography angiography and would have been apparent to the astute radiologist. Finally, CORSA and aplasia or agenesis of the ICA are rare anomalies. Though many of these cases can be asymptomatic, these anomalies have important implications in the setting of central venous line placements, surgery, thromboembolic disease, and the detection of congenital heart disease and cerebral aneurysms. Knowledge of these anomalies is important as they have a known association with cerebral aneurysms $[2,3]$, and their recognition is important for the optimization of patient care and the prevention of unnecessary invasive procedures.

\section{Statement of Ethics}

The patient has given her informed consent, and the study protocol has been approved by the institute's committee on human research.

\section{Disclosure Statement}

The authors certify that they have no affiliations with or involvement in any organization or entity with any financial interest (such as honoraria; educational grants; participation in speakers' bureaus; membership, employment, consultancies, stock ownership, or other equity interest; and expert testimony or patentlicensing arrangements) or nonfinancial interest (such as personal or professional relationships, affiliations, knowledge, or beliefs) in the subject matter or materials discussed in this paper.

\section{References}

1 Purkayastha S, Gupta AK, Varma DR, Bodhey NK, Vattoth S: Absence of the left common carotid artery with cervical origin of the right subclavian artery. Am J Neuroradiol 2006;27:708-711.

2 Amer S: Rare case of congenital absence of left internal carotid artery. Ann Ind Acad Neurol 2015;18:128-129.

3 Patel SB, Hashmi ZA, Smaroff GG, Cardone JC, Yoon PD: Congenital absence of the left carotid artery. Ann Vasc Surg 2010;24:415.e9-415.e11. 